\title{
Gain enhancement of microstrip patch antenna with via holes structure
}

\author{
Ninh Dang-Duy ${ }^{\text {a) }}$, Nanae Yoon ${ }^{\text {b) }}$, and Chulhun Seo ${ }^{\text {c) }}$ \\ Information and Telecommunication Engineering, Soongsil University, \\ 369 Sangdo-Ro, Dongjak-Gu, Seoul, Korea (06978) \\ a)duyninhbk@gmail.com \\ b) 1142014@naver.com \\ c)chulhun@ssu.ac.kr
}

Abstract: Microstrip patch antenna (MPA) with loading of shorting pins expressed as a high gain and planar structure. The inductive effect of shorting pin tunes up the resonant frequency leading to increase the electrical size of antenna and, in consequence, its radiation directivity is enhanced. This work proposes a new configuration of MPA with loading of via holes to achieve gain enhancement. The field distribution is analyzed to optimize position of via holes. A prototype of proposed MPA is fabricated and shows excellent realized gain of $11.3 \mathrm{dBi}$ that enhance about by $1.3 \mathrm{~dB}$ in comparison to the previous work.

Keywords: microstrip patch antenna, gain enhancement, shorting via holes Classification: Antennas and Propagation

\section{References}

[1] R. Q. Lee and K.-F. Lee, "Experimental study of the two-layer electromagnetically coupled rectagular patch antenna," IEEE Trans. Antennas Propag., vol. 38, no. 8, pp. 1298-1302, Aug. 1990. DOI:10.1109/8.56971

[2] D. Li, Z. Szabo, X. Qing, E.-P. Li, and Z. N. Chen, “A high gain antenna with an optimized metamaterial inspired superstrate," IEEE Trans. Antennas Propag., vol. 60, no. 12, pp. 6018-6023, Dec. 2012. DOI:10.1109/TAP.2012.2213231

[3] C. A. Balanis, Antenna Theory: Analysis and Design, John Wiley \& Sons, New Jersey, 2005.

[4] X. Zhang and L. Zhu, "High-gain circularly polarized microstrip patch antenna with loading of shorting pins," IEEE Trans. Antennas Propag., vol. 64, no. 6, pp. 2172-2178, Jun. 2016. DOI:10.1109/TAP.2016.2552544

[5] P. Bahramzy, O. Jagielski, and G. F. Pedersen, "Thermal loss and soldering effect study of high-Q antennas in handheld devices," 2013 7th European Conf. on Antennas and Propag. (EuCAP), Gothenburg, Sweden, pp. 878-881, 13597678, Apr. 2013.

[6] A. Carvalho, N. Carvalho, P. Pinho, A. Georgiadis, and A. Constanzo, " $5.8 \mathrm{GHz}$ microstrip antennas and array for microwave power transfer," 2015 9th European Conf. on Antennas and Propag. (EuCAP), Lisbon, Portugal, pp. 1-5, 15416863, Apr. 2015. 


\section{Introduction}

Simple and planar structures, low cost, and good integration are the outstanding advantages of microstrip patch antennas (MPAs); however, their low gain is the inherent drawback which prevents itself from many applications. This drawback comes mainly from the limitation of radiating plane which must satisfy the condition of resonant frequency. Generally, it can be classified into two groups of method to overcome this limitation. Firstly, the radiating plane role is taken over by adding external structures such as parasitic patches [1], or metamaterial slab [2], while the primary patch only take a role as an excited source. These techniques has been proved efficiency of gain enhancement; however, their disadvantages are considerly increasing the volume and complexity of the structure, resulting in increased simulation time and product cost. Secondly, the radiating area of MPA is directly enlarged by arraying a lot of patch elements [3], or loading a few shorting pins within the antenna's boundaries to tune up resonant frequency [4]. Antenna array is the good technique to enhance the gain but its drawbacks are notably enlarge area of antenna and require a complex feeding network. With objectives of enhancing radiating gain as well as remaining good characteristics of MPA, the technique of loading a few shorting pins is the proper selection.

In this work, the shorting pins are replaced by via holes which have the same shunt inductive effect. Synonymously, the soldering step as well as its unwanted effect in fabrication of antenna is neglected. The effect from soldering on the antenna efficiency was investigated on the inverted $\mathrm{F}$ antenna (IFA) at $740 \mathrm{MHz}$ with different soldering position at short of the IFA [5]. By experiment, this work shows the effect of soldering with shorting pins inside boundary of antenna at microwave frequency. In addition, based on Zhang's work [4], the electromagnetic fields on patch of MPA with via holes are analyzed. Two sets of via holes are inserted to center region of patch to increase the perturbation of electric field beneath the patch. The sets of via holes in square more tune up resonant frequency leading to enlarge the electrical size of this MPA and enhance radiation directivity. While the set of via holes in center line improve the reflection coefficient of MPA. The $5.8 \mathrm{GHz}$ MPA in two models of Zhang's and novel configurations were simulated and measured to validate the effectiveness of the proposed structure.

\section{MPA with loading of via holes}

Our work concentrates to design a MPA element for $5.8 \mathrm{GHz}$ microwave power transmission [6] including transmitter antenna array and receiver single antenna. Both of transmitter and receiver require the single MPA element with high gain and compact size. The high frequency laminate substrate of Taconic TLY with thickness of $1.52 \mathrm{~mm}$ is utilized to reduce the dielectric loss of the MPA.

\subsection{Shorting pin and via hole}

Fig. 1(a) shows the electric field distributions beneath the patch of conventional patch and Zhang's configuration with shorting pins and via holes. The shunt inductive effect of via hole is same as the shorting pin that notably reduces the electric field around them. Consequently, the field distribution is strongly perturbed 


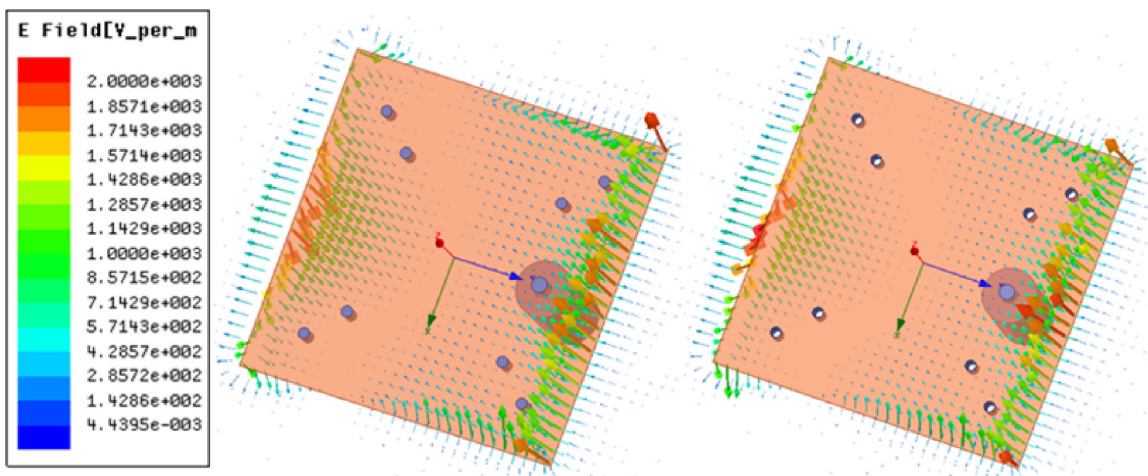

(a)

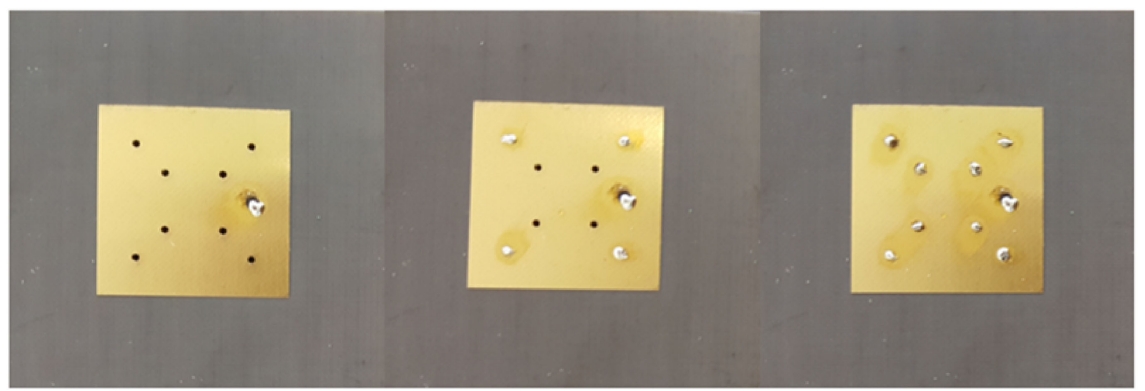

(b)

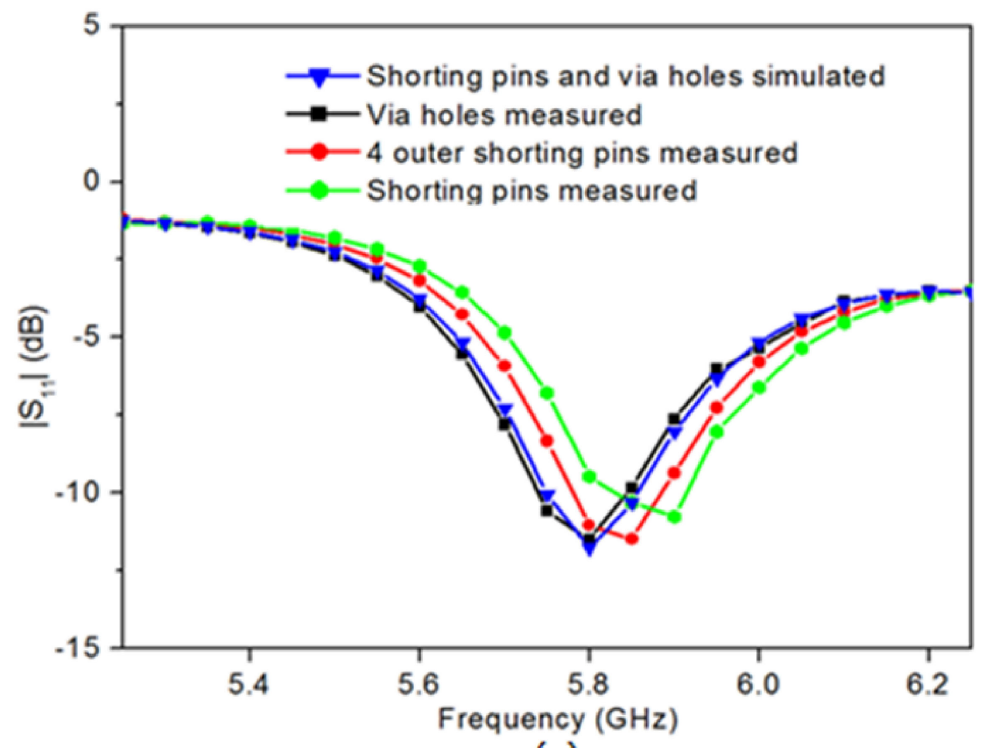

(c)

Fig. 1. Patch antennas with loading of shorting pin and via hole. (a) Electric field distributions. (b) Prototypes. (c) Reflection coefficient results.

that leads to tune up of its resonant frequency, resulting in enlarging the electrical size of patch and enhancing the radiation directivity. In fabrication, the shorting pin structure needs the soldering step to short pin between patch and ground. The addition of soldering material causes the change of resonant frequency as well as the thermal loss that heavily depends on the soldering technique. Whereas, this step can be neglected by the via hole configuration. To compare performance of two antenna, the via hole patch was fabricated and measured. Then, the pins were respectively inserted to the 4 outer and all of holes to verify the soldering effect. 
The fabricated antennas and their simulated and measured S-parameters are illustrated in Fig. 1(b)(c), respectively. The via hole antenna shows good agreement of simulated and measured results. For shorting pin antenna, the more number of pins are inserted, the more increments of resonant frequency and reflection coefficient causing by soldering become.

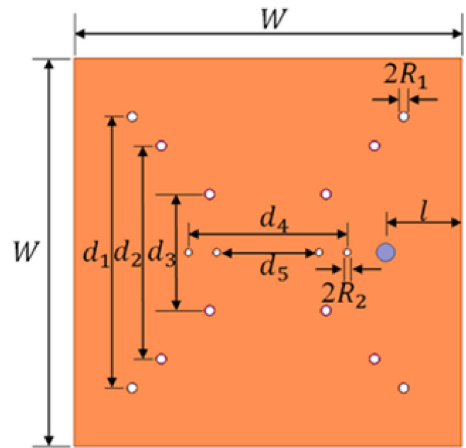

(a)
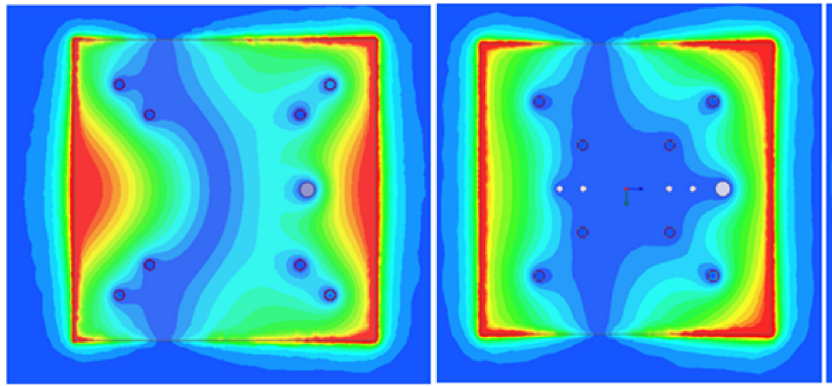

(b)

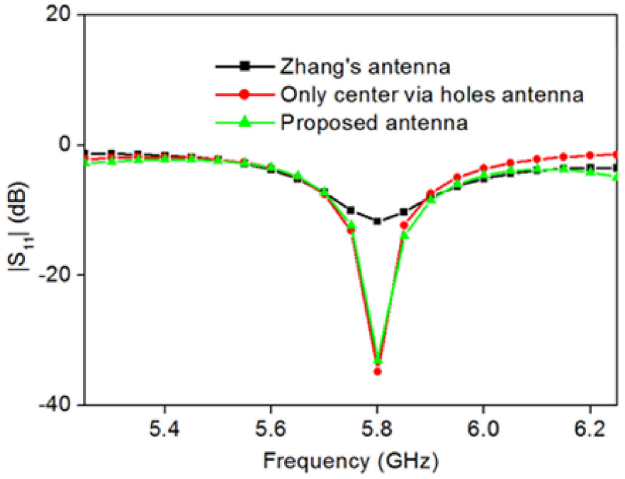

(c)

\begin{tabular}{|c|l|}
\hline$W$ & $30.3 \mathrm{~mm}$ \\
\hline$d_{1}$ & $21.2 \mathrm{~mm}$ \\
\hline$d_{2}$ & $16.7 \mathrm{~mm}$ \\
\hline$d_{3}$ & $9.1 \mathrm{~mm}$ \\
\hline$d_{4}$ & $12.4 \mathrm{~mm}$ \\
\hline$d_{5}$ & $8.0 \mathrm{~mm}$ \\
\hline$R_{1}$ & $0.5 \mathrm{~mm}$ \\
\hline$R_{2}$ & $0.3 \mathrm{~mm}$ \\
\hline$l$ & $6.0 \mathrm{~mm}$ \\
\hline
\end{tabular}
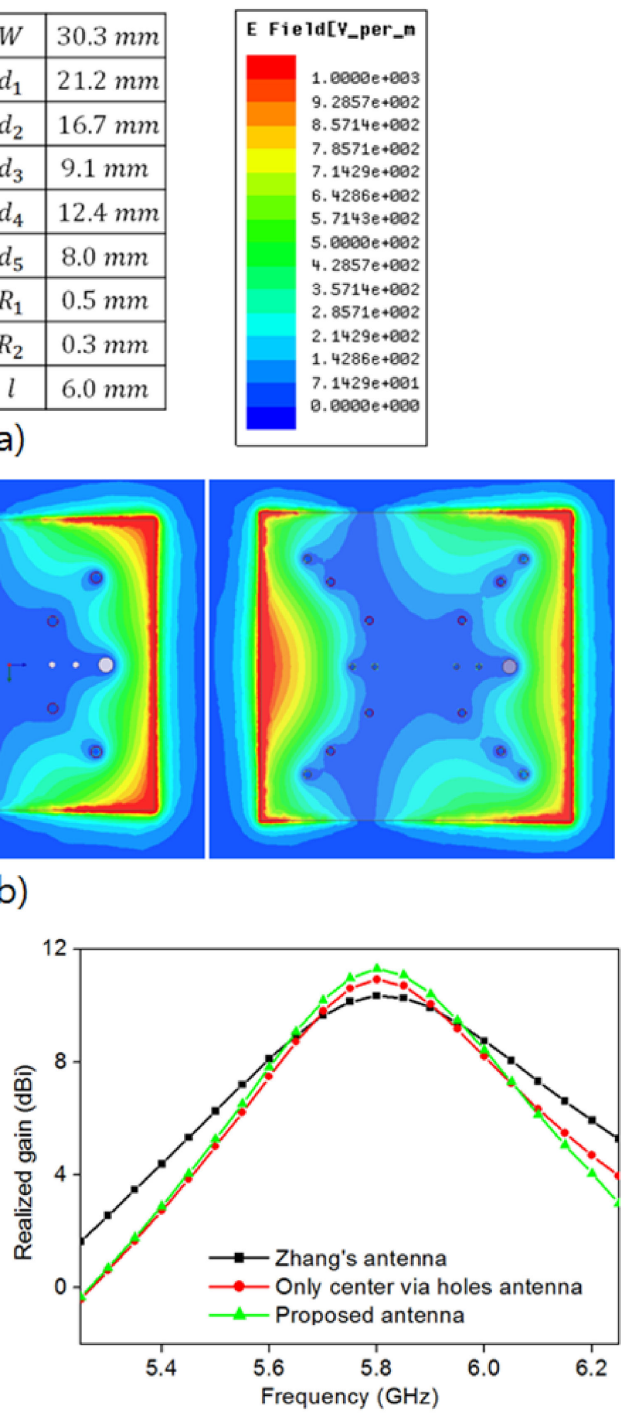

(d)

Fig. 2. Proposed antenna. (a) Antenna configuration. (b) E-field distributions. (c) Reflection coefficient results. (d) Realized gain versus frequency

\subsection{Optimal via hole configuration}

Based on Zhang's antenna [4], the novel optimal configuration of square patch with loading of via holes is proposed, as illustrated in Fig. 2(a). Three sets of via holes, with radius of $R_{1}$, are symmetrically placed along the diagonals of the patch, and each of them is placed in a square contour. From outside to inside, the width of these square contour are $d_{1}=0.7 \mathrm{~W}, d_{2}=0.55 \mathrm{~W}$, and $d_{3}=0.3 \mathrm{~W}$, respectively, where $W$ is the width of square patch. Two pairs of via holes, with radius of $R_{2}$, are 


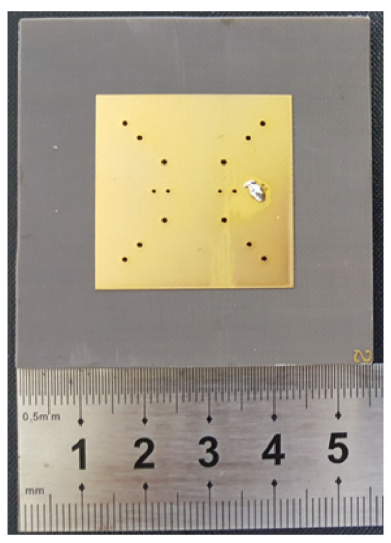

(a)

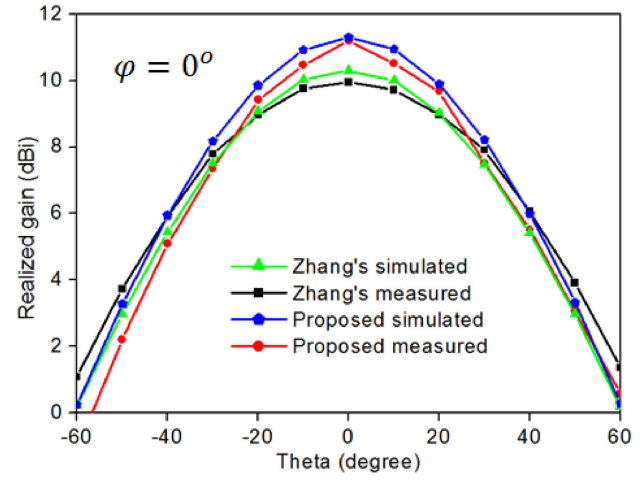

(c)

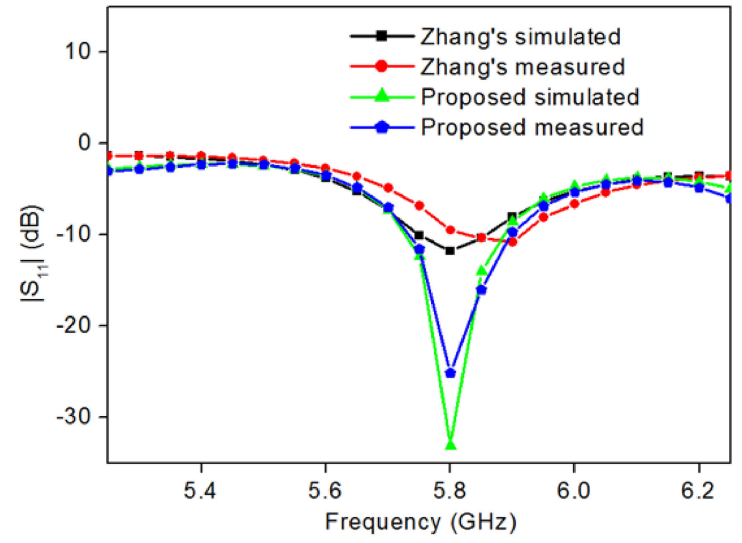

(b)

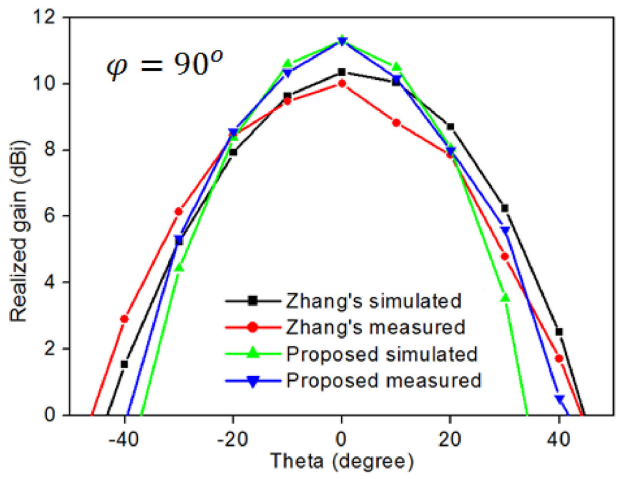

(d)

Fig. 3. Simulated and measured results. (a) Proposed antenna prototype. (b) Reflection coefficient. (c) Realized gain with $\varphi=0^{\circ}$. (d) Realized gain with $\varphi=90^{\circ}$

placed symmetrically along the middle line of the patch, which also pass through the feeding point. From outside to inside, the distance between each pair of via holes are $d_{4}=0.4 \mathrm{~W}$ and $d_{5}=0.26 \mathrm{~W}$, respectively.

Form left to hand respectively, Fig. 2(b) shows the electric field distribution of Zhang's configuration, Zhang's configuration plusing via holes along the middle line, and proposed configuration to highlight the effectiveness of extra via holes. By adding the via holes along the middle line, the electric field around the middle region of patch significantly reduces that increases the perturbation of field distribution beneath the patch. In addition, optimizing position of the center via holes also improves the match between feeding port and patch, as expressed by the reflection coefficient in Fig. 2(c). Compare to Zhang's configuration, the antenna with center via holes obtain gain enhancement of $0.6 \mathrm{~dB}$. In proposed model, a set of 4 via holes placing in a square contour with the width of $d_{3}$ are inserted to optimize performance of antenna. A quite perturbation of electric field distribution is achieved leading to enhance realized gain of $0.4 \mathrm{~dB}$, as shown in Fig. 2(d).

To verify the proposed antenna, a prototype was fabricated as shown in Fig. 3(a). The overall size of ground plane is $55.0 \times 55.0 \mathrm{~mm}$ satisfying compactness condition for microwave power transmission application as well as minimizing the diffraction from finite ground and its effect on radiation patterns. The simulated and measured reflection coefficients of Zhang's and proposed antenna are plotted in Fig. 3(b) which shows the good agreement of simulated and measured results of 
proposed antenna. Fig. 3(c)(d) respectively plot the simulated and measured realized gain of the proposed and Zhang's antennas in the two principal planes, i.e., $\varphi=0^{\circ}$ and $\varphi=90^{\circ}$. The proposed antenna with via holes configuration presents an excellent agreement between simulated and measured results. In simulation, the gain enhancement of proposed antenna is only $1 \mathrm{~dB}$; however, in measurement, the gain enhancement of proposed antenna is $1.3 \mathrm{~dB}$.

\section{Conclusion}

In this paper, an optimal configuration of microstrip patch antenna with loading of via hole has been proposed to achieve gain enhancement. The antenna with via holes neglects the soldering step in conventional work and shows the good agreement results between simulation and measurement. By adding two sets of via holes to center region of patch, the perturbation of electric field is increased that is a key to enhance realized gain. The optimal position of via holes in the middle line of patch also improves the reflection coefficient. The prototype of proposed antenna was fabricated and measured that indicates the gain enhancement of $1.3 \mathrm{~dB}$.

\section{Acknowledgement}

This work was supported by the National Research Foundation of Korea (NRF) grant funded by the Korean government (MSIP) (NRF-2017R1A5A1015596). 